ARTICLE

DOI: $10.1038 / \mathrm{s} 41467-018-06619-\mathrm{x}$

\title{
Spatially-resolved fluorescence-detected two-dimensional electronic spectroscopy probes varying excitonic structure in photosynthetic bacteria
}

\author{
Vivek Tiwari (10 1, Yassel Acosta Matutes ${ }^{2}$, Alastair T. Gardiner ${ }^{3}$, Thomas L.C. Jansen (10 ${ }^{4}$, \\ Richard J. Cogdell (i) ${ }^{3} \&$ Jennifer P. Ogilvie (iD ${ }^{1}$
}

Conventional implementations of two-dimensional electronic spectroscopy typically spatially average over $\sim 10^{10}$ chromophores spread over $\sim 10^{4}$ micron square area, limiting their ability to characterize spatially heterogeneous samples. Here we present a variation of twodimensional electronic spectroscopy that is capable of mapping spatially varying differences in excitonic structure, with sensitivity orders of magnitude better than conventional spatiallyaveraged electronic spectroscopies. The approach performs fluorescence-detection-based fully collinear two-dimensional electronic spectroscopy in a microscope, combining femtosecond time-resolution, sub-micron spatial resolution, and the sensitivity of fluorescence detection. We demonstrate the approach on a mixture of photosynthetic bacteria that are known to exhibit variations in electronic structure with growth conditions. Spatial variations in the constitution of mixed bacterial colonies manifests as spatially varying peak intensities in the measured two-dimensional contour maps, which exhibit distinct diagonal and crosspeaks that reflect differences in the excitonic structure of the bacterial proteins.

\footnotetext{
${ }^{1}$ Department of Physics, University of Michigan, Ann Arbor, Michigan 48109, USA. ${ }^{2}$ Applied Physics Program, University of Michigan, Ann Arbor, Michigan 48109, USA. ${ }^{3}$ Institute for Molecular Biology, University of Glasgow, Glasgow G12 8TA, UK. ${ }^{4}$ Zernike Institute for Advanced Materials, University of Groningen, Groningen 48105, The Netherlands. Correspondence and requests for materials should be addressed to J.P.O. (email: jogilvie@umich.edu)
} 
U ltrafast spectroscopy ${ }^{1}$, has contributed to the fundamental understanding of a wide range of processes including the primary steps of vision ${ }^{2}$, energy transfer, and charge separation in natural ${ }^{3}$ and artificial light-harvesting systems, and carrier relaxation pathways in semiconductors ${ }^{4-6}$. Understanding the ultrafast electronic dynamics between excited electronic states of molecules is important in many fields, ranging from artificial $^{7-9}$ and natural light harvesting ${ }^{10}$ to the chemistry of melanin pigments at cancerous tumor sites ${ }^{11}$. Although pumpprobe spectroscopies can capture ultrafast relaxation processes, important information such as broadening mechanisms and couplings between excited electronic states that underlie ultrafast processes are obscured in such measurements ${ }^{12}$.

The invention of two-dimensional electronic spectroscopy $(2 \mathrm{DES})^{12}$, which is an optical analog of $2 \mathrm{D}$ nuclear magnetic resonance $(\mathrm{NMR})^{13}$, has provided high experimental frequency resolution sufficient to distinguish many similar transient chemical species based on their correlated absorption and subsequent emission properties. By correlating the initial absorption frequencies of a system with subsequent changes along a detection frequency axis, a $2 \mathrm{D}$ contour map of highly resolved excitation and detection frequency axes can decongest the couplings that cause transitions between electronic states, to the extent that is limited only by the molecular resolution. Due to the added dimensionality, the information content of a 2DES experiment is a superset of what is available from pump-probe spectroscopy. 2DES experiments have been broadly applied, and have contributed to the mechanistic understanding of delocalized electronic and mixed electronic-vibrational states in natural photosynthetic systems ${ }^{14-18}$, molecular aggregates ${ }^{19}$, hybrid organic/inorganic perovskites ${ }^{8,9}$, and singlet fission materials ${ }^{20}$.

Despite its success in advancing fundamental understanding of a variety of condensed phase phenomena, most 2DES experiments have offered no spatial resolution (typically a few hundreds of microns). This limitation produces 2DES signals that represent an ensemble-averaged response over a large number of species. Many of the condensed phase systems for which 2DES experiments can provide the deepest insight possess highly disordered spatial and/or energetic landscapes, rendering many experimental conclusions susceptible to ensemble averaging effects. For instance, in photosynthetic systems, where the protein environment imparts disorder in pigment electronic excited state energies, coherent dynamics between such states will "artificially" damp because the measured 2DES response will be a weighted average over the entire disordered energetic landscape. Such "ensemble dephasing" has been experimentally observed between a pair of excitons in a photosynthetic protein ${ }^{21,22}$. A timeresolved molecular level understanding of the role of morphology on the performance of a number of artificial photovoltaic materials is also limited due to spatial averaging. For instance, heterogeneous domain composition exists in polymer/fullerene solar cells and modulates exciton dissociation and charge recombination on picosecond timescales ${ }^{23,24}$. Similarly, the effect of grain size $^{25}$ and chloride content ${ }^{26}$ on charge transport in perovskites thin-films is well known, but the connections between layer heterogeneity and charge delocalization is poorly understood. Spatial-resolution provided by pump-probe microscopy has partly addressed these questions ${ }^{11,27-29}$. However, new spatially resolved tools that can go beyond pump-probe approaches to resolve inhomogeneity and the couplings between excited electronic states directly with high frequency and time-resolution could impact our understanding of a broad range of systems, including emerging $2 \mathrm{D}$ materials ${ }^{30}$.

Here, we present spatially resolved fluorescence-detected twodimensional electronic spectroscopy (SF-2DES), which combines the femtosecond time resolution of a broadband laser pulse and the frequency resolution of 2D spectroscopy with spatial resolution beyond that of a two-photon microscope. The use of rapid phase-modulation and lock-in detection enables the use of high repetition rate lasers compatible with imaging applications and produces high signal-to-noise ratio images. We demonstrate in vivo measurements on a mixture of photosynthetic bacterial cells from Rps. palustris grown under different light intensity conditions. SF-2DES reveals spatially varying excitonic structure (that manifest as distinct 2D peaks) as a function of spatial heterogeneity in the constitution of the bacterial colony. By adopting a fluorescence-detection approach, as opposed to radiated electric-field detection in conventional space-averaged 2DES measurements, we estimate nearly six orders of magnitude fewer bacterial cells contribute to the signal compared to conventional 2D measurements on similar systems ${ }^{31}$. We show that our measurements can differentiate between the growth-conditiondependent perturbations in the excitonic structure of constituent bacteria with a high signal-to-noise ratio.

\section{Results}

Methodology. A 2D experiment ${ }^{32}$ is comprised of three pulses with precisely controlled time-spacing between the pulses. Each pulse interacts with the sample up to $1^{\text {st }}$ order in time-dependent perturbation theory. When there is a manifold of coupled excited electronic states, the effect of the first two "pump" pulses can be understood as preparing a superposition of excited (or ground) states, which is then allowed to evolve during the time interval between the second and third pulses, typically called the pumpprobe waiting time. The third pulse probes the evolving superposition by generating a $3^{\text {rd }}$ order macroscopic polarization in the sample at different pump-probe waiting times. In the presence of an ensemble of dipoles, the fields radiated by individual oscillating dipoles within the macroscopic polarization coherently add up to generate an experimentally detectable electric field signal along a specific phase-matched direction, which depends on a linear combination of the wavevectors of the individual pulses, that is, $\mathbf{k}_{\mathrm{sig}, \mathrm{R}}=-\mathbf{k}_{1}+\mathbf{k}_{2}+\mathbf{k}_{3}$ and $\mathbf{k}_{\mathrm{sig}, \mathrm{NR}}=+\mathbf{k}_{\mathbf{1}}-\mathbf{k}_{2}+\mathbf{k}_{3}$, where $\mathbf{k}_{\mathrm{sig,R}}$ and $\mathbf{k}_{\mathrm{sig,NR}}$ are rephasing and non-rephasing $2 \mathrm{D}$ signals, and $\mathbf{k}_{\mathrm{i}}, i=1-3$ are wavevectors of the three pulses. Phase-matching allows background-free detection, providing a route towards high signal-to-noise ratios. However, phase-matching also constrains the $2 \mathrm{D}$ experiment. The generation of a phase-matched signal intrinsically relies on probing dipole ensembles with volumes larger than $\lambda^{3}$, where $\lambda$ is the wavelength of light. In addition, background free detection through phase-matching requires that the pulses be non-collinear, making the experiment difficult to integrate with a microscope objective, thus constraining the possibility of spatially resolved measurements.

Fluorescence detected two-dimensional electronic spectroscopy (F-2DES) in a fully collinear geometry was first implemented by Warren and co-workers using a phase-cycled acousto-optic modulator (AOM) based pulse-shaping approach ${ }^{33}$. Fluorescence detection implies that sample volumes smaller than $\lambda^{3}$ can be detected as the need for a macroscopic grating of dipoles radiating a detectable electric field is replaced by the detection of fluorescence from an excited state population that is produced by the addition of a $4^{\text {th }}$ pulse. A fully collinear geometry implies that the setup can be integrated with a microscope objective, making spatially resolved measurements facile. In the fully collinear geometry, background-free detection can be achieved by replacing phase-matching with phase-cycling ${ }^{33}$ or phasemodulation $^{34}$. Several phase-cycling approaches to F-2DES have been demonstrated ${ }^{33,35,36}$. To date, phase-cycling methods have been employed in spatially resolved 2D-infrared (2DIR) vibrational spectroscopy in the ground electronic state of a 
metal-carbonyl stained polystyrene bead ${ }^{37,38}$. Very recently, Goetz et al. ${ }^{39}$ performed phase-cycling-based F-2DES in a microscope. Some of the phase-cycling-based approaches that have been used for imaging applications have relied on a single AOM pulse-shaper to create multiple time-delayed pulses. Depending on the pulse-shaper, this may constrain the method to only a few tens of $\mathrm{kHz}$ laser repetition rates. In addition, control over the polarization and spectrum of each pulse, both of which have been routinely exploited to suppress unwanted signals $^{32}$, may become difficult to implement. While better for use with high repetition rate lasers, spatial-light-modulator pulseshapers have relatively slow switching times, making setups that employ them susceptible to laser noise. In addition, phase-cycling approaches often require that as many as 27 scans with different relative pulse phases be acquired to extract a single absorptive $2 \mathrm{D}$ spectrum $^{35,39}$. For samples where photobleaching is significant, this requirement is particularly problematic.

Instead of phase-cycling, we adopt the alternative phasemodulation approach to F-2DES demonstrated by Marcus and co-workers $^{34}$ for our implementation of SF-2DES. A simplified layout of the setup is shown in Fig. 1. Four time-delayed pulses are created using interferometers, MZ1 and MZ2, and the carrierenvelope phase of each pulse, $\Omega_{\mathrm{i}}$ is scanned by its respective
AOM, over the laser pulse train. Thus, each pulse is tagged with a unique radio-frequency $\Omega_{\mathrm{i}}$, which replaces the unique wavevectors, $\mathbf{k}_{\mathrm{i}}$ of a $2 \mathrm{DES}$ experiment. The resulting rephasing and nonrephasing $2 \mathrm{D}$ signals are contained in a four-wave mixing (FWM) population which modulates at the linear combination of radio frequencies of the individual pulses, that is, $\Omega_{\mathrm{R}}=-\Omega_{1}+\Omega_{2}+$ $\Omega_{3}-\Omega_{4}$ and $\Omega_{\mathrm{NR}}=\Omega_{1}-\Omega_{2}+\Omega_{3}-\Omega_{4}$, for rephasing and nonrephasing signals, respectively. The two signals are demodulated and detected in parallel lock-in channels using phase-sensitive lock-in detection ${ }^{40}$. The signal is physically undersampled through detection relative to a reference wavelength of $826 \mathrm{~nm}$, generated from REF1 and REF2 outputs of MZ1 and MZ2, respectively (see Methods, Supplementary Figure 2 for details). The reference frequencies are chosen close to the electronic energy gap, such that the signal phase oscillates at $\left(\omega_{\text {eg }}-\omega_{\mathrm{R} 1(2)}\right)$, where $\omega_{\text {eg }}$ is the electronic energy gap which is sampled during time delay $t_{1(3)}$, and $\omega_{\mathrm{R} 1(2)}$ are reference frequencies generated using REF1(2). Undersampling makes the measurement insensitive to phase noise caused by mechanical delay fluctuations in the interferometer arms, thus avoiding the need for active-phase stabilization $^{41}$. In contrast to the approaches mentioned above, there is no constraint of $\mathrm{kHz}$ pulse repetition rates, and the polarization and spectrum of each pulse can be independently

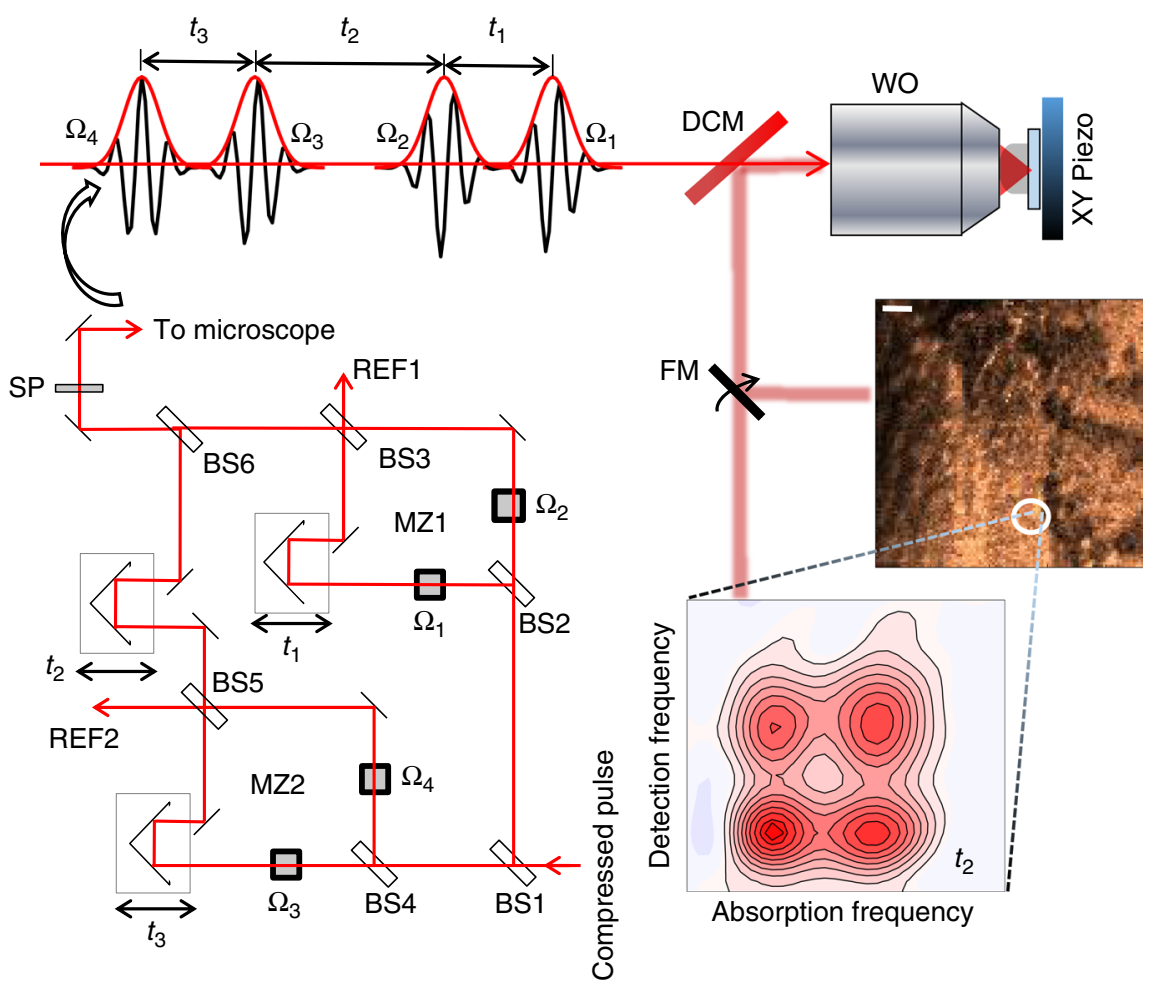

Fig. 1 Spatially resolved fluorescence-detected 2DES spectrometer (SF-2DES). Further details of the spectrometer are provided in Supplementary Figure 2. A given pulse in the compressed laser pulse train is split 50:50 by a beamsplitter (BS1), and each half is routed into a Mach-Zehnder (MZ) interferometer (MZ1 and MZ2). Each of the four interferometer arms (two per MZ) contains an AOM which sweeps the carrier-envelope phase of the pulse by frequency $\Omega_{\mathrm{i}}, i=1-4$. The time intervals between the four pulses, $t_{1}, t_{2}$, and $t_{3}$ are controlled by mechanical delay stages. One output port from each $M Z$ is used to generate a reference signal REF1(2), which is utilized by the lock-in amplifier for signal detection. The other output port from each MZ is combined at BS6, generating four collinear time separated pulses (pump and probe pulse pairs), which are optically filtered by a shortpass filter (SP), and routed into a confocal microscope. A dichroic mirror (DCM) in the microscope transmits the collinear pulse train towards a water objective (WO), which focuses it on an immobilized sample. The sample is mounted on an XY scanning piezo stage (PZ). The fluorescence collected by the WO is separated from the excitation light at the DCM, and can be either routed for fluorescence imaging, or for generating a 2D map. An example of the fluorescence image, and the $2 \mathrm{D}$ spectrum at a desired $\mathrm{XY}$ location is shown in the figure. The $2 \mathrm{D}$ spectrum corresponds to zero waiting time between pump and probe pulse pairs $\left(t_{2}=\right.$ 0 ), and shows absorptive changes in the refractive index of the sample in the form of distinct $2 \mathrm{D}$ peakshapes. Cross peaks at $t_{2}=0$ indicate that the absorption and detection frequencies of the system are different. This implies that the transitions corresponding to the positions of the two diagonal peaks correspond to excitonic transitions between sites which are electronically coupled on the excited state, and therefore connected via a common ground electronic state, and a common doubly excited electronic state manifold 
controlled with ease. Use of a lock-in amplifier for phase-sensitive signal detection is another major advantage that allows high signal detection sensitivity over a wide dynamic range. Modulation of the resulting signal at high frequencies implies that $1 / f$ noise can be minimized, and frequency filtering by lock-in detection enables minimization of white noise. Moreover, phasing of the $2 \mathrm{D}$ signal is done in the time-domain at $t_{1}, t_{2}, t_{3}=0$ by the lock-in amplifier, making it substantially easier than many other approaches to $2 \mathrm{DES}^{32}$. Importantly, in the phase-modulation approach $^{34}$, the phase-cycling happens in "real time" as each AOM sweeps the carrier-envelope phase over the laser pulse train. This obviates the need in the phase-cycling approach $33,35,36,39$ for combining multiple separate phase scans during which time photobleaching and laser fluctuations will reduce the signal-tonoise ratio with which the desired signal can be extracted.

To perform SF-2DES, the collinear phase-modulated pulse train generated by MZ1 and MZ2 is routed to a confocal microscope as shown in Fig. 1. First a fluorescence map of the sample is generated, followed by acquisition of F-2DES spectra at the desired XY locations.

SF-2DES on unmixed samples. Chromatic adaptation in a number of species of purple photosynthetic bacteria involves both a growth in the size of the photosynthetic unit through an increase in the number of peripheral light-harvesting antenna complexes (LH2) per reaction center core (RC-LH1 complex $)^{42}$, as well as synthesis of LH2 complexes with modified spectral properties ${ }^{43}$. Under high light (HL) conditions the LH2 complex contains a monomeric ring of 9 bacteriochlorophyll a $(B C h l a)$ pigments which absorbs at $\sim 800$ $\mathrm{nm}$ (B800 ring), and a dimeric ring of $18 \mathrm{BChl} a$ pigments which absorbs at $\sim 850 \mathrm{~nm}$ (B850 ring) at $300 \mathrm{~K}$. The pigments are held together by nine $\alpha \beta$ polypeptide pairs whose composition is lightintensity-dependent, and is dictated by which of the multigene family of puc genes are expressed ${ }^{44}$. Rps. palustris presents an interesting case because under lower light (LL) intensity conditions, the B850 ring loses oscillator strength and a band around $810 \mathrm{~nm}$ appears. This has been attributed to the polypeptide inhomogeneity within an LH2 ring, causing a blue-shift in the site energies of certain BChl a pigments ${ }^{45}$.

Figure 2 shows in vivo measurements illustrating how the growth-condition-dependent perturbation of the electronic structure of purple bacteria manifests in the SF-2DES spectra. Figure 2a shows fluorescence images collected from a bacterial colony in unmixed samples of LL (upper panel) and HL (lower panel) bacteria. Multiple XY locations on these fluorescence maps were chosen to collect fluorescence-detected 2DES spectra. Figure $2 \mathrm{~b}$ shows the averaged absorptive fluorescence detected 2DES spectra for LL and HL bacteria. The positions of the two diagonal peaks correspond to the B800 (upper diagonal) and B850 (lower diagonal) excitonic manifolds of the LH2 antennae within the a

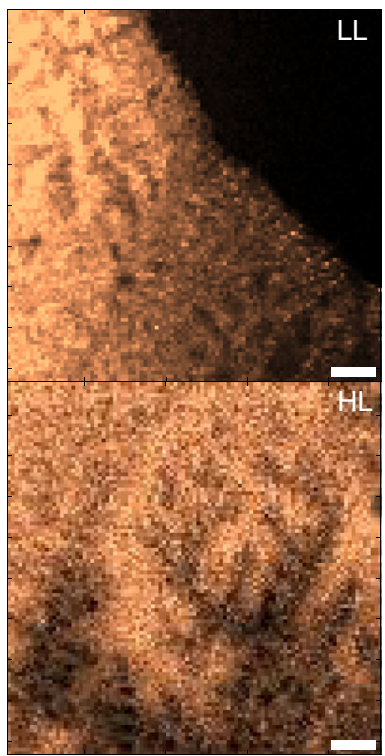

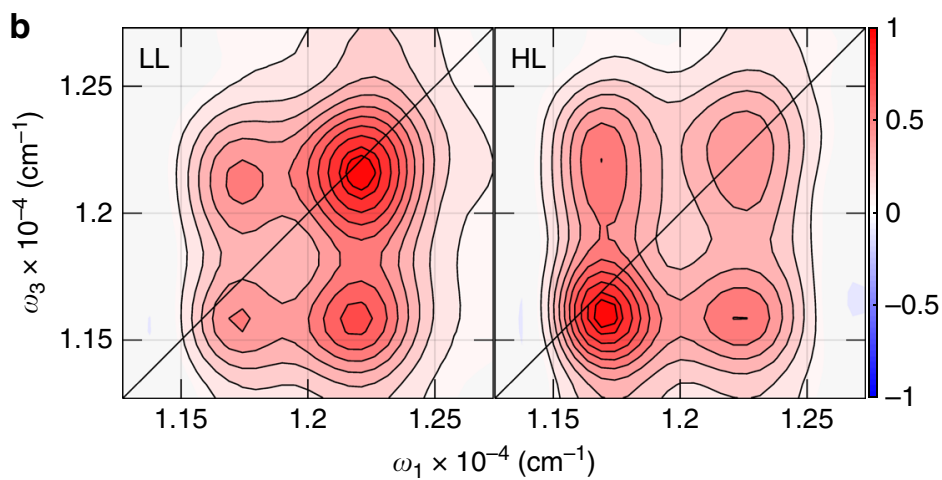

C

Wavenumbers $\times 1 \mathrm{E}-4(\mathrm{~cm}-1)$

d

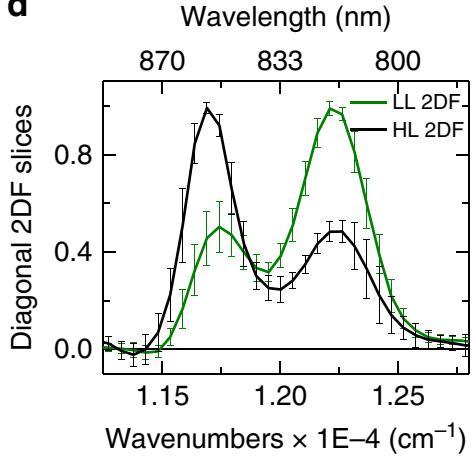

Fig. 2 SF-2DESon unmixed cells of Rps. palustris grown under high and low light (HL and LL). a Confocal fluorescence images of a drop-dried film of photosynthetic bacteria Rps. palustris. The bacteria were grown under LL (top panel), and HL (bottom panel) conditions. A $10 \mu \mathrm{m}$ scale bar is shown for reference. The OD of the live cell solution from which the samples were prepared is shown in panel (c) and the drop volume was measured to be $\sim 0.08 \mu \mathrm{L}$ through gravimetric analysis (Supplementary Note 3, Supplementary Figure 7). $\mathbf{b}$ Absorptive 2D spectra at $t_{2}=0$ fs obtained by averaging 2D spectra from 5 different $X Y$ locations on the $\mathrm{LL}$ and $\mathrm{HL}$ fluorescence images, as shown in the left and right panels, respectively. The spectra show distinct cross-peaks at $t_{2}=0 \mathrm{fs}$. The LL and HL 2D spectra are normalized relative to the B800 and B850 bands, respectively, to more clearly emphasize their spectral differences. Contours are drawn at $10-90 \%$ in steps of $10 \%$, with additional contours at 95 and $100 \%$ to highlight small differences in maxima. The frequency $\omega$ corresponding to the axes labels on the 2D plots corresponds to $\omega=\left|\omega^{\prime}\right| / 2 \pi c$, where $\omega^{\prime}$ is in rad/fs. c Linear absorption spectrum for LL and HL grown Rps. palustris overlaid with the laser spectrum (gray area). The OD for the LL sample is scaled by a factor of 0.37 such both samples have the same OD at the B800 band. Note that the LL and HL cell concentrations are different. $\mathbf{d}$ Slices through the maxima of the upper and lower diagonal peaks of the LL and HL 2D spectra shown in panel $\mathbf{b}$. The error bars are obtained from averaging $L L$ and $H L 2 D$ spectra at 5 different locations on the fluorescence images of panel a. The solid black line across the $2 \mathrm{D}$ plot corresponds to the diagonal. All measurements were conducted at $300 \mathrm{~K}$ 
bacterial cells. The effect of growth conditions on spectral properties is reflected by the varying strength of the lower (B850) diagonal peak. This is better seen in Fig. 2d, which compares slices through the maxima of the diagonal peaks for the LL and HL cases. The measurements show that between HL and LL conditions, the 2D diagonal peak strengths change by a factor of four, that is, the ratio of B850/B800 diagonal peak changes from 2:1 to 1:2. The measured changes are well above the error bars of the measurement, emphasizing that the SF-2DES spectrometer is able to distinguish between the two kinds of cells with a respectable signal-to-noise ratio. Higher error bars near the peak slopes in Fig. $2 \mathrm{~d}$ reflect the fact that any index shifts in the $2 \mathrm{D}$ spectra, resulting from trial-to-trial variations, are averaged over. Averaging the $2 \mathrm{D}$ spectra would also lead to broader than ideal 2D peakshapes. The strength of a $2 \mathrm{D}$ peak is a product of the absorption and emission transition strengths ${ }^{12}$, such that it depends on $\sim \mu^{4}$, where $\mu$ is the electronic transition dipole magnitude. Thus, a factor of 4 change on the diagonal peaks from HL and LL cells suggests that the absorption strength $\left(\mu^{2}\right)$ for the B850 manifold decreases by a factor of 2 under low light (LL) conditions. The cross-peak strengths are less perturbed than the diagonal peaks because they depend on the product of B800 and B850 absorption strengths. The 2D cross-peaks also highlight the fact that despite the strong perturbation of the site energies of the $B C h l a$ pigments on the $\mathrm{B} 800$ and $\mathrm{B} 850$ rings, the $B C h l a$ transition dipoles at B800 and B850 sites remain coupled through Coulomb interactions. Figure $2 \mathrm{c}$ compares the absorption spectrum of HL and LL grown cells. It is seen that the spectral changes highlighted by the absorption spectrum, which reflect the strength $\mu^{2}$ of a given electronic transition, are less than that indicated by the $2 \mathrm{D}$ peaks because, as mentioned above, the $2 \mathrm{D}$ peak strengths depend on $\mu^{4}$, and thus are more sensitive to changes in the excitonic structure. Both LL and HL absorption spectra show a shoulder around 875 $\mathrm{nm}$ (B875 band), which corresponds to the absorption of the LH1 complex. The laser spectrum (shown as solid gray area) overlaps dominantly with the blue part of the B850 band, and excitation of the $\mathrm{B} 875$ band is minimal.

SF-2DES on mixed samples. The measurements discussed above establish that spectral differences in the LL and HL cells can be clearly differentiated by a SF-2DES experiment. In order to demonstrate the spatial-resolution and ability to characterize complex samples provided by the SF-2DES spectrometer, we performed measurements on bacterial colonies with a spatially heterogeneous composition of the LL and HL cells. The idea behind these measurements was to simulate what one might expect in a heterogeneous thin film sample, such as those known in mixed halide perovskites $28,29,46$, and polymer-fullerene blends $^{23}$. In a mixed sample, the overall $2 \mathrm{D}$ signal from a given point in space will be averaged over the HL and LL constituent cells. In order to deduce the ratio of HL:LL cells contributing to the $2 \mathrm{D}$ signal, the averaged HL and LL spectra in Fig. $2 \mathrm{~b}$ are chosen as the $2 \mathrm{D}$ basis spectra, and a linear least squares fit of the mixed 2D signal is performed. Such an analysis assumes that the HL and LL cells are equally fluorescent. Thus a given HL:LL ratio indicates the ratio of FWM signal from HL versus LL cells, rather than the ratio of the number of HL to LL cells. Supplementary Figure 14 presents a possible calibration scheme for calibrating the measured FWM signal to number of cells.

Figure 3a shows the confocal fluorescence image obtained from a bacterial colony which has spatially heterogeneous composition of HL and LL cells. A 2D spectrum was collected at several points on this image, marked in red squares. The points are separated horizontally by $\sim 5 \mu \mathrm{m}$. Three such $2 \mathrm{D}$ spectra, from the locations corresponding to the red, blue, and green solid dots, are shown in
Fig. 3b. A fit of the 2D spectrum from each location as a linear combination of the $2 \mathrm{D}$ basis spectra is shown in the middle frame of Fig. 3b, and the residual is shown in the right frame. The plots for all other locations, and the fitting details, are provided in Supplementary Figures 3-5. The three chosen locations approximately show the extremes of the expected spatial variations, that is, a dominantly HL (middle) or LL (bottom) spectrum, as well as a spectrum which is an equal mixture (top). The fits and the residuals are shown on the same scale as the data, and show average fit errors of $\sim 10 \%$, across all the locations marked in red squares in Fig. 3a, emphasizing the capability of SF-2DES to spatially resolve the excitonic structure differences manifested in 2D peakshapes and amplitudes, into their heterogeneous constituents. Morphological variations in the pump-probe signal are already known for a number of systems $s^{11,23,28,29}$. However, in terms of resolving such variations into $2 \mathrm{D}$ cross-peaks so as to infer electronic transitions connected through a common ground state, the capability provided by SF-2DES is highly complementary to conventional 2DES.

Figure $3 c$ shows the LL and HL 2D spectra from unmixed sample solutions of live cells which were recirculated using a peristaltic pump. These spectra are considered "ideal cases" $\left(\mathrm{LL}_{\mathrm{i}}\right.$ and $\left.\mathrm{HL}_{\mathrm{i}}\right)$ because they exhibit no photobleaching artifacts. Changes in the sample OD and FWM signal before and after these experiments were not measurable beyond trial-to-trial variations. Normally, Mie scattering encountered in flowing similar colloidal solutions 31,47 becomes a dominant source of noise in interferometric detection. However, despite the all-collinear geometry, the combination of spectrally separating the fluorescence from the laser, and highfrequency lock-in detection, allows us to make in vivo measurements with relative ease. A comparison of $\mathrm{LL}_{\mathrm{i}}$ and $\mathrm{HL}_{\mathrm{i}}$ spectra to the basis $2 \mathrm{D}$ spectra in Fig. $2 \mathrm{~b}$, and with the $2 \mathrm{D}$ spectra on mixed samples (Fig. 3b), shows vertical distortions in the spectra collected from immobilized samples. This distortion is partly caused by FWM signal degradation due to photobleaching of $B C h l$ a pigments within the cells (see Discussion).

SF-2DES theoretical resolution. A diffraction-limited optical imaging system images an ideal point as a three-dimensional light intensity distribution also called the point spread function (PSF) 48. For a conventional fluorescence imaging system, the PSF is defined as $H_{\mathrm{id}, \text { conv }}=I(r, z)$, where $I(r, z)$ denotes the excitation light intensity distribution at the focus, and the subscript "id" denotes that it is an ideal diffraction-limited PSF. Here $r$ and $z$ are lateral and axial coordinates, respectively ${ }^{48}$. For confocal microscopy, the PSF depends linearly on the excitation intensity, as well as on the size of a point detector, and is given by $H_{\mathrm{id}, \mathrm{conf}}(r, z)=I$ $(r, z)[I(r, z) \otimes D(r)]$. The second term results from a twodimensional convolution of the excitation intensity with a detector $D(r)$, such as a pinhole, and is responsible for optical sectioning in confocal microscopy. When the pinhole is large compared to the size of the Airy disc, the second term becomes constant, such that $H_{\text {id,conf }}$ is approximately the same as conventional PSF $H_{\mathrm{id}, \text { conv }}$, that is, $H_{\mathrm{id}, \text { conf }}(r, z) \sim H_{\mathrm{id}, \text { conv }}{ }^{48}$. This is also true for the confocal imaging part of the current experiment because a large detection pinhole $(200 \mu \mathrm{m}$ pinhole diameter compared to a sub-micron Airy disc diameter) renders the effect of a detection pinhole on the confocal PSF negligible.

Assuming a large detection pinhole, for the case of two-photon (TP) microscopy, the excitation point spread function (PSF) depends quadratically on the excitation intensity, that is, $H_{\mathrm{id}, \mathrm{TP}}(r, z) \sim H_{\mathrm{id}, \mathrm{conf}}^{2}(r / 2, z / 2)=I^{2}(r / 2, z / 2)^{48}$. The functional dependence of intensity on $r, z$ is different in the TP case due to the difference of $1 / 2$ in the excitation wavelength. In analogy, the FWM signal in a fluorescence-detected 2DES experiment is 
a

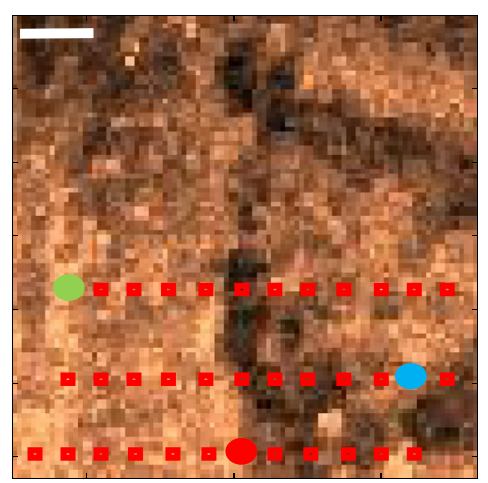

C

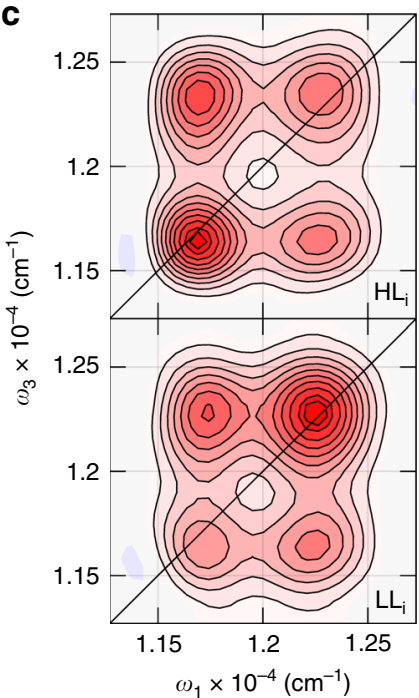

b
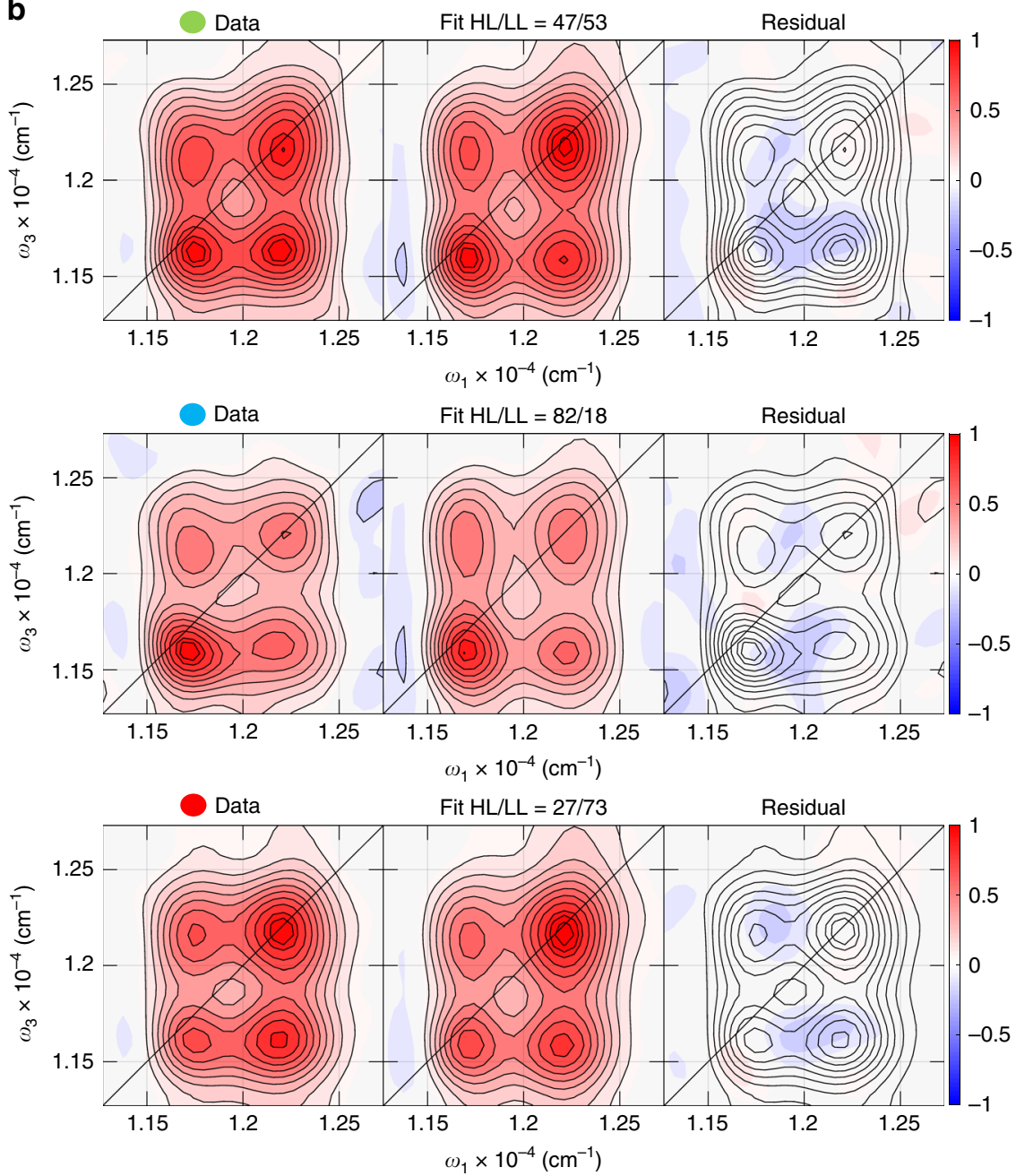

Fig. 3 SF-2DES on mixed cells of Rps. palustris grown under high and low light (HL and LL). a Confocal fluorescence image from a mixed, LL and HL, dropdried film of photosynthetic bacteria Rps. palustris. Red squares correspond to all the locations where a 2D spectrum was collected, and is shown in Supplementary Figures 3-5. The red, blue and green solid dots indicate locations for which the corresponding $2 \mathrm{D}$ spectra are shown in panel b. A $10 \mu \mathrm{m}$ scale bar is shown for reference. $\mathbf{b}$ Normalized $t_{2}=0 \mathrm{fs}$ absorptive $2 \mathrm{D}$ spectra collected at the three solid dot locations shown on the fluorescence image in panel a. The three horizontal frames show the measured 2D spectrum (left frame), a linear least squares fit obtained by using the LL and HL 2D spectra (in Fig. 2b) as the basis spectra to describe the spectrum collected from a mixed sample (middle frame), and the resulting residual (right frame). All the frames in a panel are normalized to the left frame. The middle frame also displays the ratio of purely HL and LL 2D basis spectra (shown in Fig. 2b), which best fits the measured spectrum. c Absorptive $t_{2}=0 \mathrm{fs} 2 \mathrm{D}$ spectra for the $\mathrm{HL}$ and $\mathrm{LL}$ samples obtained by recirculating a live cell solution through a $200 \mu \mathrm{m}$ pathlength sample cell using a peristaltic pump. These spectra represent the ideal scenario (represented as $\mathrm{LL}_{i}, \mathrm{HL}_{\mathrm{i}}$ ), where there is no measurable photobleaching. The OD of the sample in $1 \mathrm{~mm}$ pathlength corresponds to that shown in Fig. 2c. Contours are drawn at $10-90 \%$ in steps of $10 \%$, with additional contours at 95 and 100\% to highlight small differences in maxima. The frequency $\omega$ corresponding to the axes on the 2D plots corresponds to $\omega=\left|\omega^{\prime}\right| / 2 \pi c$, where $\omega$ is in rad/fs. The solid black line across the $2 \mathrm{D}$ plot corresponds to the diagonal. All measurements were conducted at $300 \mathrm{~K}$

generated by one light-matter interaction of the sample with each pulse in sequence of pump and probe pulse pairs, and therefore depends linearly on the pump and probe intensities. Thus, the volume of the FWM excitation PSF, which dictates the volume of the sample contributing to the FWM signal, will be a product of pump and probe intensities, that is, $H_{\mathrm{id}, \mathrm{FWM}}(r, z) \sim I^{2}(r, z)$. Note that unlike in the case of TP microscopy, the FWM process and fluorescence detection happens at approximately similar wavelengths, and therefore, $r, z$ do not have a factor of $1 / 2$. Consequently, the spatial resolution dictated by $H_{\mathrm{id}, \mathrm{FWM}}$ will be better than that provided by TP microscopy.

For the water objective (NA 1.2) used for SF-2DES experiments at a peak laser wavelength of $\sim 820 \mathrm{~nm}$, the ideal FWM PSF $H_{\mathrm{id}}$, $\operatorname{FWM}(r, z)$ is shown in Fig. 4, left panel. The lateral and axial FWHMs from Fig. 4, left panel are $\sim 0.25 \mu \mathrm{m}$ and $\sim 0.69 \mu \mathrm{m}$, respectively. These represent the diffraction-limited spatial resolution obtainable from a SF-2DES spectrometer. As with confocal TP microscopy ${ }^{48}$, the use of a smaller pinhole could further improve the spatial resolution of SF-2DES.

Experimental determination of SF-2DES resolution and sensitivity. In order to estimate the sensitivity of SF-2DES compared to conventional heterodyne-detected 2DES studies, we perform an order of magnitude estimation of the number of cells contributing to the FWM signal measurements on bacterial colonies, and compare to recent in vivo studies ${ }^{31,47}$ on cells using conventional $2 \mathrm{DES}$.

A confocal fluorescence image of a $0.5 \mu \mathrm{m}$ bead measured with the imaging part of the SF-2DES spectrometer shows an average FWHM lateral diameter of $0.83 \mu \mathrm{m}$. The fluorescence image, 


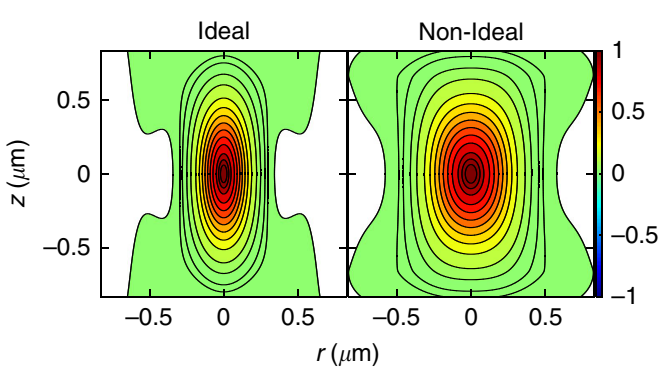

Fig. 4 Ideal and experimental four-wave mixing (FWM) point spread function (PSF). (left panel) The expected ideal diffraction-limited FWM PSF for the case when the pump-probe laser beams with $820 \mathrm{~nm}$ peak laser wavelengths, are focused to a point in the sample using a water objective of NA 1.2, and a peak laser wavelength of $820 \mathrm{~nm}$. (right panel) The non-ideal FWM PSF calculated from the ideal FWM PSF by convoluting it with a 0.54 $\mu \mathrm{m}$ diameter bead to approximate the measured experimental broadening. Contours are drawn at $0.1 \%, 1 \%, 2 \%, 5 \%$, and $10-90 \%$ in steps of 10,95 , and $98 \%$

fitting, and PSF estimation details are provided in Supplementary Note 4 and Supplementary Figures 8-10. However, the expected FWHM, obtained by convoluting the diffraction-limited lateral confocal PSF with a $0.5 \mu \mathrm{m}$ bead, is $\sim 0.52 \mu \mathrm{m}$. This implies that the experimentally broadened lateral confocal PSF deviates from the ideal case by a factor of $\sim 0.83 / 0.52=1.6$. Based on this deviation, in order to estimate the experimental non-ideal FWM PSF, we first convolute the ideal confocal PSF $H_{\text {id,conf, with a bead }}$ of a given diameter, such that the resulting confocal PSF has a lateral width of $1.6 \times$ the ideal lateral width. A bead diameter of $0.54 \mu \mathrm{m}$ approximates this experimental broadening, and the resulting non-ideal confocal PSF is denoted as $H_{\text {nid,conf, where the }}$ subscript "nid" denotes the non-ideal case. The non-ideal FWM PSF $H_{\text {nid,FWM }}$ is then calculated as $H_{\text {nid,FWM }} \sim H_{\text {nid,conf }}^{2}$. Figure $4 \mathrm{~b}$ shows the estimated $H_{\text {nid,FWM }}$, which dictates the experimental spatial resolution. As discussed above, the FWM PSF in Fig. 4 (right panel) approximates the experimental lateral broadening of the PSF measured through the confocal image. We note that the above analysis assumes that the experimental broadening of the diffraction-limited PSF along the axial direction is also affected in the same way as measured along the lateral direction, which is a reasonable assumption for an order of magnitude estimation.

The volume enclosed by the $10 \%$ contour in the FWM PSF in Fig. 4, defines the region inside which the excitation probability of a FWM signal is $>10 \%$ of the maximum excitation probability in the experiment, that is, the sample volume from which $>90 \%$ of the FWM signal contributes. For the ideal case in the left panel, the enclosed $90 \%$ volume is $\sim 0.19 \mu \mathrm{m}^{3}$, and increases to $\sim 0.55 \mu \mathrm{m}^{3}$ (0.55 femtoliters) when the FWM PSF is non-ideal (right panel). In comparison, in a conventional 2DES experiment with a $200 \mu \mathrm{m}$ pathlength and a $100 \mu \mathrm{m}$ diameter spot size, the pump-probe overlap volume is $\sim 1.6 \times 10^{6} \mu \mathrm{m}^{3}$, which is over six orders of magnitude larger than in our SF-2DES setup. Assuming the same starting OD for the experiments, we estimate $\sim 25$ cells $/ \mu \mathrm{m}^{3}$ of the solution. This gives an upper estimate of $\sim 10^{3}$ cells in a dried drop of $\sim 0.08 \mu \mathrm{L}$ initial volume contributing to the FWM signal in SF-2DES. This estimate is detailed in Supplementary Note 3.

\section{Discussion}

A reduction in the probed volume by approximately six orders of magnitude of SF-2DES compared to conventional 2DES is due to the optical sectioning enabled by the FWM PSF of the microscope. As discussed above, the lateral and axial resolution provided by nonlinear imaging through SF-2DES will be better than two-photon imaging, and could be further enhanced with a smaller pinhole for confocal detection ${ }^{48}$. Further resolution enhancement could be possible with the use of metallic nanostructures, which have served as optical antennas ${ }^{49}$ that allow sub-diffraction-limited focussing of electromagnetic fields. Optical antennas have allowed near-field imaging with femtosecond lasers with spatial resolution down to a few tens of nanometers ${ }^{50,51}$. Plasmonic enhancement also allows detection of weak FWM signals, even reaching the single-molecule $\operatorname{limit}^{51}$. Similar resolution of a few tens of nanometers has also been demonstrated in variation of stimulated emission depletion microscopy (STED) with picosecond time resolution ${ }^{52}$. The collinear geometry, facile manipulation of the spectra of individual pulses, and frequency filtering by the lock-in amplifier makes it possible to integrate the SF-2DES spectrometer with the above super-resolution imaging approaches. Moreover, the collinear geometry reduces distortions of the $2 \mathrm{D}$ peakshapes due to directional filtering and phasematching ${ }^{53}$. For applications involving optically thick samples, such as deep tissue imaging, resonant fluorescence re-absorption, which is not an issue in TP microscopy, could cause higher order non-linear effects such as sequential and parallel signal cascades ${ }^{54,55}$, or absorptive/dispersive signal distortions ${ }^{53}$.

A comparison of Figs. $2 \mathrm{c}$ and $3 \mathrm{~b}$ with Fig. $3 \mathrm{c}$ shows that the ideal $\left(\mathrm{LL}_{\mathrm{i}}\right.$ and $\left.\mathrm{HL}_{\mathrm{i}}\right)$ 2D spectra are distorted vertically when measured on immobilized samples. This vertical broadening is in part due to averaging the spectra over multiple locations on the fluorescence image, but a majority can be accounted for by FWM signal degradation due to photobleaching. Experimentally, the $t_{1}$ time delay is scanned for each successive value of $t_{3}$. Thus, a $t_{3}$ slice (corresponding to $t_{1}=0 \mathrm{fs}$ ) is acquired over a longer time period during which photobleaching can accumulate, leading to the vertical distortion along $\omega_{3}$. Assuming an exponential signal degradation with each successive time index in the experiment, a simulation of this effect (shown in Supplementary Figures 1 and 12) captures the observed 2D peak broadening due to photobleaching. The simulations show that photobleaching as much as $80 \%$ of the signal during the data collection time of $45 \mathrm{~s}$ causes $2 \mathrm{D}$ peaks to merge together, with cross-peaks appearing as shoulders. Based on the distinct peaks seen in Fig. 3b, we estimate an upper limit of $60 \%$ signal degradation during the data collection time. Signal degradation is expected to effect dynamics along the pump-probe waiting time $t_{2}$, however the $2 \mathrm{D}$ cross-peak features probed at $t_{2}=0 \mathrm{fs}$, remain surprisingly robust. Improvements such as larger time steps made possible by physical undersampling of the signal, that is, signal detection at $\omega_{\mathrm{eg}}-\omega_{\mathrm{R} 1(2)}$ frequency, can easily reduce the experiment time by order $N^{2}$, where $N$ is the total number of time steps in the experiment. For example, in the current experiment, using $t_{1}$ and $t_{3}$ time steps of $10 \mathrm{fs}$ instead of $5 \mathrm{fs}$, reduces the data collection time to $\sim 12 \mathrm{~s}$ without any frequency aliasing effects (see Supplementary Figure 6). Reduced dimensionality experiments that extract particular signals of interest along the waiting time ${ }^{56}$, can also lead to substantially faster data collection and improved signalto-noise ratios. Additionally, greater sample preparation efforts to reduce photobleaching, by using oxygen scavengers or low temperature could reduce spectral distortions due to photobleaching. Lower laser repetition rates, down to $\sim 1 \mathrm{MHz}$, are also known to reduce photobleaching and increased fluorescence signal levels ${ }^{57}$. We note that although we observe photobleaching effects, our phase-modulation-based SF-2DES approach should be considerably less susceptible to distortions from photobleaching than phasecycling methods. Since the phase-cycling approach relies on the acquisition of 27 consecutive scans that are combined to extract the $2 \mathrm{D}$ spectrum $^{39}$, to achieve a high signal-to-noise ratio it is critical that the signal level be stable throughout all 27 scans to enable effective signal isolation. We note that, as in conventional 2DES, the laser spectrum influences $2 \mathrm{D}$ peak amplitudes and must be considered for quantitative measurements as discussed in Supplementary Note 10 and Supplementary Figures 15-16. 
The background-free nature of fluorescence-detection, coupled with the development of high quantum efficiency single-photon detectors has made single-molecule fluorescence detection routin ${ }^{58}$. Thus, in terms of scalability of 2DES to very low numbers of absorbers, fluorescence-detection approach may hold more promise than heterodyne-detection of a radiated electric field, as typically employed in ensemble 2DES experiments. Our experiments demonstrate that F-2DES is highly sensitive: for experiments in which the sample was recirculated (Fig. 3c), a respectable signal-to-noise ratio was attainable with ODs $\sim 5 \times$ lower (in a $200 \mu \mathrm{m}$ pathlength) than those used in previous in vivo studies ${ }^{31,47}$, with the estimated probed volume $\sim 6$ orders of magnitude smaller due to the FWM PSF created by the microscope objective. Varying energy transfer pathways have been reported ${ }^{59}$ for isolated LH2 complexes through singlephoton counting. SF-2DES offers a promising route towards observing such effects using $2 \mathrm{D}$ spectroscopy, and resolving the debate ${ }^{10}$ surrounding the timescale of the survival of coherent wavepackets through sub-ensemble resolution.

Conventional 2D measurements of detergent-isolated LH2 complexes show very weak (and sometimes negative) cross-peaks at $t_{2}=$ $0 \mathrm{fs}^{60,61}$, in contrast to strong, positive cross-peaks in fluorescencedetected spectra. Such differences in cross-peak structure between conventional and fluorescence-detected 2D measurements have been reported in simulations of a dimer by Lott et al. ${ }^{62}$, who attribute the effect to differences in the relative signs of signal contributions in the two experiments that lead to different addition/cancellation effects. Recently, Tokmakoff and co-workers have also reported similar differences $^{63}$ in fluorescence-encoded 2DIR measurements. This issue is discussed in further detail in Supplementary Note 11 and Supplementary Figure 17. In other photosynthetic antennas, conventional 2D measurements with specific polarization schemes have revealed ${ }^{64}$ the early waiting time positive cross-peaks not seen in $2 \mathrm{D}$ measurements with all parallel polarization scheme. The distinct $t_{2}=0 \mathrm{fs}$ cross-peaks in our data suggest the B800 and B850 transitions possess a common ground state, consistent with previous pump-probe studies $43,65,66$. Supplementary Note 12 and Supplementary Figures 18-19 present a simplified exciton dimer model for estimation of Coulomb couplings and transition dipole strengths from 2D peaks. Further theoretical work is needed to properly weight the different cross-peak signal contributions and to understand what quantitative measurements can be derived from present F-2DES studies. We note that during revision of our manuscript, Pullerits and co-workers reported fluorescence-detected 2D spectra of detergent-isolated $\mathrm{LH} 2{ }^{67}$, showing similar prominent cross-peaks. They propose that the prominence of the cross-peaks reveals a considerably larger degree of delocalization between B800 and B850 rings than was previously assumed. We note that in some systems high repetition rate excitation can lead to an accumulation of excited states that could distort 2D spectra. We discuss this issue in detail in Supplementary Note 13 and Supplementary Figures 20-23, where we argue that such contributions are minimal in our measurements, and also present experiments employing $500 \mathrm{kHz}$ excitation (Supplementary Figure 13) that are in good agreement with our data. In future applications, complementary approaches such as pump-probe spectroscopy or conventional 2DES, as well as modifications of this experiment that cancel out the $2 \mathrm{D}$ signal for $t_{2}<0 \mathrm{fs}$ via phase-cycling approaches ${ }^{39}$, can accurately quantify the extent of the build-up of stationary states and their effect on the SF-2DES spectra, beyond what is possible through qualitative modeling of photo-physics.

For non-fluorescent samples, the SF-2DES spectrometer can be modified for $2 \mathrm{D}$ photocurrent detection ${ }^{68}$, or for heterodyne detection by delaying the $4^{\text {th }}$ pulse and routing it around the sample as the local oscillator ${ }^{69}$. Domain heterogeneity in polymer-fullerene blends, or thin films of perovskites and TIPSpentacene, could also quench the photoluminescence at certain locations in the film ${ }^{70}$, making the above modification necessary in order to probe the domain-dependent carrier delocalization dynamics in these materials that has been observed in pumpprobe microscopy studies $23,27,28$.

In conclusion, we have demonstrated in vivo spatially resolved measurements of $2 \mathrm{D}$ electronic spectra from colonies of photosynthetic bacteria grown under different light intensity conditions. While the current spatial resolution of our SF-2DES approach cannot resolve spatially heterogeneous electronic structure within a single bacterium, the high signal-to-noise ratio of the data from stationary and circulating cells demonstrates that the approach enables in vivo studies that can capture changes in excitonic structure due to growth-condition-dependent remodeling of the photosynthetic membrane. The changes in excitonic structure in the B850 ring due to growth conditions are reflected as spatially varying $2 \mathrm{D}$ peak intensities, ultimately caused by spatial heterogeneity in the bacterial colonies. 2D peaks due to intra-B850 ring couplings between the $850 \mathrm{~nm}$ and $810 \mathrm{~nm} \mathrm{BChl} \mathrm{a} \mathrm{sites} \mathrm{are} \mathrm{expected} \mathrm{to} \mathrm{be}$ weak, and are not resolved in our room temperature measurements. The fluorescence-detection approach we adopt offers significant sensitivity improvements over conventional heterodyne detection. Employing phase-modulation rather than phase-cycling enables imaging at high repetition rates, and produces spectra with a high signal-to-noise ratio without the need to combine multiple phasecycled scans that could be susceptible to photobleaching. This work serves as a proof-of-concept demonstration of the broad applicability of the SF-2DES approach. Future applications will explore the complementary nature of conventional and fluorescence-detected 2D approaches and work towards establishing connections between morphological variations, the resulting excitonic structure, and ultimately the performance of a variety of natural and artificial lightharvesting materials.

\section{Methods}

SF-2DES setup. Here we provide a concise description of SF-2DES spectrometer, shown in Fig. 1, and refer the reader to Supplementary Note 1 for additional details. The experimental layout is based on the original fluorescence-detected 2DES design by Marcus and co-workers ${ }^{34}$. The output from a commercial broadband $83 \mathrm{MHz} \mathrm{Ti}$ : Sapphire oscillator (Venteon One) is sent into an SLM-based pulse-shaper (MIIPS 640 $\mathrm{P}$, Biophotonic Solutions) for dispersion pre-compensation. Laser pulses from the pulse shaper of $\sim 20 \mathrm{fs}$ FWHM pulse duration are split by a 50:50 beamsplitter and routed into two Mach-Zehnder (MZ) interferometers. Within each of the two MZs, the pulse is further split using 50:50 beamsplitters (BS), and each arm is tagged with a unique phase that is modulated at radio frequency using AOMs. The phase modulation frequencies for the four arms are denoted by $\Omega_{1-4}$, such that when the split pulses are recombined at BS3 and BS5, within MZ1 and MZ2, respectively, the pulse amplitude modulates at the difference frequencies of the AOMs, that is, $\Omega_{12}$ and $\Omega_{34}$ for MZ1 and MZ2, respectively. One output port from BS3 and BS5 is used to generate reference frequencies REF1 and REF2 for MZ1 and MZ2, respectively, which modulate, respectively, at $\Omega_{12}$ and $\Omega_{34}$ difference frequencies. These frequencies are used for phase-sensitive lock-in detection. Beams from the other two output ports (one from each of the two MZs) are recombined at BS6 to produce a train of four collinear pulses. The relative pulse time delays are controlled by translational stages resulting in an all-collinear train of 4 pulses separated by time delays $t_{1}$ between the first two pulses, $t_{2}$ between the $2^{\text {nd }}$ and $3^{\text {rd }}$ pulses, and $t_{3}$ between pulses 3 and 4 in the sequence.

The train of 4 phase-modulated pulses passes through a OD4 875 SP filter (Edmund Optics), and is routed into a confocal microscope (Olympus $1 \times 51$ ). The pulse train is transmitted through a $875 \mathrm{~nm}$ dichroic mirror (Semrock) inside the microscope before reaching the water objective (Olympus PlanApo 60x, NA1.2) with the objective collar set to 0.17 . The immobilized sample is mounted on an XY scanning piezo stage (Piezo Instruments, P-612.2SL). The fluorescence is collected in the epi-detection geometry and directed by a DCM to be spatially filtered through a $200 \mu \mathrm{m}$ detection pinhole, followed by optical filtering through an OD 4 875LP filter. For linear fluorescence imaging experiments, the filtered fluorescence is focused onto a single-photon counting APD1 (not shown in Fig. 1). The photon counts registered by APD1 are counted using a counting circuit (not shown in Fig. 1) for each XY position of the computer controlled piezo controller.

For SF-2DES measurements on the imaged sample, or for fluorescence detected 2DES experiments in which the sample is circulated, the filtered fluorescence is routed by a flip mirror (FM) and focused on another APD operating in linear mode (not shown in Fig. 1). $t_{1}$ and $t_{3}$ delays are scanned from 0 to $90 \mathrm{fs}$ in steps of $5 \mathrm{fs}$, and $t_{2}$ delay is scannable from 0 to $800 \mathrm{ps}$, but is fixed at $t_{2}=0 \mathrm{fs}$ for the experiment. The FWM signal generated by the sample oscillates at the difference frequencies $\left(\Omega_{3}-\Omega_{4}\right) \pm$ 
$\left(\Omega_{1}-\Omega_{2}\right)$, where the (positive) negative sign corresponds to (non-rephasing) rephasing $2 \mathrm{D}$ signals. The oscillating signal is demodulated using a lock-in amplifier (Zurich Instruments, HF2LI). Physical undersampling of the oscillating signal, as well as phasesensitive detection, is achieved by signal detection relative to the reference signals generated using REF1 and REF2 MZ ports. The reference signals are connected to the lock-in amplifier parallel reference channels corresponding to rephasing and nonrephasing signals. The references for all the SF-2DES experiments are set at $826 \mathrm{~nm}$. The AOM frequencies are set at $\Omega_{1}=80.111 \mathrm{MHz}, \Omega_{2}=80.101 \mathrm{MHz}, \Omega_{3}=80.029$ $\mathrm{MHz}$, and $\Omega_{4}=80.0 \mathrm{MHz}$ through a common clock, such that the resulting signals oscillate at $19 \mathrm{kHz}$ (rephasing) and $39 \mathrm{kHz}$ (non-rephasing). The data collection time for each 2D spectrum was $\sim 45 \mathrm{~s}$ for $5 \mathrm{fs} t_{1,3}$ steps. The data collected for each $t_{2}$ delay, is zero-padded upto 128 points, and Fourier transformed with respect to $t_{1}$ and $t_{3}$, resulting in a correlated map of absorption $\left(\omega_{1}\right)$ and detection $\left(\omega_{3}\right)$ frequency axes as shown in Fig. 1. Raw data without zero-padding is presented in Supplementary Figure 11. A flip mirror (FM) is used to switch between the imaging and spectroscopy modalities. A 2D spectrum can thus be generated for all the desired points on the fluorescence image.

For experiments where the sample is circulated, a $200 \mu \mathrm{m}$ pathlength sample cuvette and an air objective (Olympus LUCPlanFLN 40×, NA0.6) are used for the measurements. The sample is circulated through the cuvette using a peristaltic pump at flow rates of $190 \mathrm{ml} / \mathrm{min}$. For linear fluorescence imaging, the total incident power on the sample is $\sim 0.125 \mu \mathrm{W} /$ pulse, corresponding to a fluence of $0.26 \mu \mathrm{J} / \mathrm{cm}^{2} /$ pulse. The binning time at each piezo location is $5 \mathrm{~ms}$. For SF-2DES experiments on immobilized and circulating samples, the spectra were collected with a total power of $7.5 \mu \mathrm{W} / \mathrm{pulse}$, corresponding to a total fluence of $12-16 \mu \mathrm{J} /$ $\mathrm{cm}^{2} /$ pulse. At $83 \mathrm{MHz}$ repetition rate, the total pulse energies for these fluences is $\sim 0.09 \mathrm{pJ} /$ pulse, such that the excitation probability at the center of the pulse is less than $0.1 \%$. Low excitation probability is chosen so as to minimize multiple excitations on the molecule by the same pulse.

Sample preparation and handling. Cells of Rps. palustris strain 2.1.6 were grown anaerobically in closed, flat-sided bottles using C-succinate media at $30{ }^{\circ} \mathrm{C}$ under anaerobic conditions at different light intensities. HL cultures were placed between two $100 \mathrm{~W}$ incandescent bulbs to give either HL (HL, $\sim 150 \mu$ mol photons s$~^{-1} \mathrm{~m}^{-2}$ ) and LL (LL, $\sim 100 \mu \mathrm{mol}$ photons $\mathrm{s}^{-1} \mathrm{~m}^{-2}$ ). The cells were harvested when fully adapted, washed in $20 \mathrm{mM}$ MES, $100 \mathrm{mM} \mathrm{KCl}, \mathrm{pH} 6.8$, aliquoted, flash frozen and, stored at $-20^{\circ} \mathrm{C}$ until required. For the experiments, a thick aliquot of cells was suspended in the buffer such that the final OD in a $1 \mathrm{~mm}$ cuvette was 0.38 for $\mathrm{HL}$ and 1.03 for LL cells. Using the microscope eye piece, it was checked that the cells in a drop of a diluted sample solution were motile. No oxygen scavenging agents were used in the cell solutions. For experiments with circulating sample, $\sim 10 \mathrm{ml}$ of starting cell solution was circulated through a $200 \mu \mathrm{m}$ cuvette. During the experiment, the sample was kept in an ice-cooled water bath using a Peltier cooler. For experiments on immobilized samples, a $0.08 \mu \mathrm{L}$ drop of the cell solution was dispensed on a $0.17 \mathrm{~mm}$ coverslip using a syringe tip (BD U-100 31G), and the drop was allowed to dry under nitrogen gas. The average volume of the syringe-dispensed drop was measured to be $0.079 \pm 0.002 \mu \mathrm{L}$ using gravimetric analysis (by measuring the average weight of drop of 15 syringedispensed drops in three trials). The coverslips were cleaned by leaving them in a bath of $6 \mathrm{M} \mathrm{HCl}$ in ethanol on a hot plate at $100^{\circ} \mathrm{C}$ for $\sim 2 \mathrm{~h}$, followed by sonication for a few hours in a bath of distilled water and ethanol. However, for the experiments on mixed HL and LL samples, the coverslips were only cleaned with distilled water such that the surface of the coverslips was hydrophobic enough to not allow the HL and LL drop to spread and mix together uncontrollably. For the mixed HL/LL experiments, initially a HL drop was allowed to dry followed by dispensing a LL drop close to the original drop. The LL drop was manually dragged (using a syringe tip) near the dried HL drop until it partially mixed with the HL drop. The final drop was allowed to dry under nitrogen, and the coverslip was sealed against a glass slide using double-sided tape on the inner surface and Scotch tape on the outer surface. The diameter of the final drop after drying was measured to be $\sim 1 \mathrm{~mm}$. Typically, the experiments were conducted within $\sim 1 \mathrm{~h}$ of sample preparation. Using the microscope eyepiece it was observed that after more than 3 weeks of drying the cells under Nitrogen, the cells regained motility once a drop of water was poured on the dried drop.

For fluorescence imaging in order to estimate the experimental confocal PSF, commercially available fluorescent polystyrene beads (Fluoresbrite 763 Carboxylate Microspheres $0.50 \mu \mathrm{m}$ ) were diluted by a factor of $10^{8}$ in distilled water, and a $20 \mu \mathrm{L}$ drop was drop-dried on a $0.17 \mathrm{~mm}$ microscope coverslip. The absorption and emission wavelength of the dye inside the beads was $763 \mathrm{~nm}$ and $820 \mathrm{~nm}$, respectively. The laser spectrum used for imaging the bead was filtered with a 800 shortpass excitation filter (Chroma), and the fluorescence was detected with a 815 longpass detection filter (Chroma). Due to the 800 shortpass filter, the peak laser excitation wavelength was $780 \mathrm{~nm}$.

\section{Data availability}

The data sets collected and analyzed for the reported experiments are available from the corresponding authors upon reasonable request.

Received: 12 February 2018 Accepted: 17 September 2018 Published online: 11 October 2018

\section{References}

1. Scherer, N. F., Knee, J. L., Smith, D. D. \& Zewail, A. H. Femtosecond photofragment spectroscopy: the reaction ICN $\rightarrow \mathrm{CN}+$ I. J. Phys. Chem. C. 89, 5141-5143 (1985).

2. Schoenlein, R. W., Peteanu, L. A., Mathies, R. A. \& Shank, C. V. The first step in vision: femtosecond isomerization of rhodopsin. Science 254, 412-415 (1991).

3. Blankenship, R. E. Molecular Mechanisms of Photosynthesis.. (Blackwell Science Ltd, Oxford, 2008).

4. Woggon, U. et al. Ultrafast energy relaxation in quantum dots. Phys. Rev. B 54, 17681-17690 (1996).

5. George, P. A. et al. Ultrafast optical-pump terahertz-probe spectroscopy of the carrier relaxation and recombination dynamics in epitaxial graphene. Nano. Lett. 8, 4248-4251 (2008).

6. Chen, Y.-C. et al. Ultrafast optical switching properties of single-wall carbon nanotube polymer composites at $1.55 \mu \mathrm{m}$. Appl. Phys. Lett. 81, 975-977 (2002).

7. Provencher, F. et al. Direct observation of ultrafast long-range charge separation at polymer-fullerene heterojunctions. Nat. Commun. 5, 4288-4298 (2014).

8. Richter, M. J. et al. Ultrafast carrier thermalization in lead iodide perovskite probed with two-dimensional electronic spectroscopy. Nat. Commun. 8, 376-382 (2017).

9. Jha, A. et al. Direct observation of ultrafast exciton dissociation in lead iodide perovskite by 2D electronic spectroscopy. ACS Photonics 5, 852-860 (2018).

10. Scholes, G. D. et al. Using coherence to enhance function in chemical and biophysical systems. Nature 543, 647-656 (2017).

11. Matthews, T. E., Piletic, I. R., Selim, M. A., Simpson, M. J. \& Warren, W. S Pump-probe imaging differentiates melanoma from melanocytic nevi. Sci. Transl. Med. 3, 71ra15-71ra24 (2011).

12. Jonas, D. M. Two-dimensional femtosecond spectroscopy. Annu. Rev. Phys. Chem. 54, 425-463 (2003).

13. Aue, W. P., Bartholdi, E. \& Ernst, R. R. Two-dimensional spectroscopy. application to nuclear magnetic resonance. J. Chem. Phys. 64, 2229-2246 (1976).

14. Engel, G. S. et al. Evidence for wavelike energy transfer through quantum coherence in photosynthetic systems. Nature 446, 782-786 (2007).

15. Fuller, F. D. et al. Vibronic coherence in oxygenic photosynthesis. Nat. Chem. 6, 706-711 (2014).

16. Womick, J. M. \& Moran, A. M. Vibronic enhancement of exciton sizes and energy transport in photosynthetic complexes. J. Phys. Chem. B 115, 1347-1356 (2011).

17. Dean, J. C., Mirkovic, T., Toa, Z. S. D., Oblinsky, D. G. \& Scholes, G. D. Vibronic enhancement of algae light harvesting. Chem 1, 858-872 (2014).

18. Paleček, D., Edlund, P., Westenhoff, S. \& Zigmantas, D. Quantum coherence as a witness of vibronically hot energy transfer in bacterial reaction center. Sci. Adv. 3, e1603141-e1603146 (2017).

19. Lim, J. et al. Vibronic origin of long-lived coherence in an artificial molecular light harvester. Nat. Commun. 6, 7755-7761 (2015).

20. Bakulin, A. A. et al. Real-time observation of multiexcitonic states in ultrafast singlet fission using coherent 2D electronic spectroscopy. Nat. Chem. 8, 16-23 (2016).

21. Savikhin, S., Buck, D. R. \& Struve, W. S. Oscillating anisotropies in a bacteriochlorophyll protein: evidence for quantum beating between exciton levels. Chem. Phys. 223, 303-312 (1997).

22. Thyrhaug, E. et al. Identification and characterization of diverse coherences in the Fenna-Matthews-Olson complex. Nat. Chem. 10, 780-786 (2018).

23. Kesava, S. V. et al. Domain compositions and fullerene aggregation govern charge photogeneration in polymer/fullerene solar cells. Adv. Energy Mater. 4, 1400116-1400125 (2014).

24. Rao, A. et al. The role of spin in the kinetic control of recombination in organic photovoltaics. Nature 500, 435-439 (2013).

25. Nie, W. et al. High-efficiency solution-processed perovskite solar cells with millimeter-scale grains. Science 347, 522-525 (2015).

26. Eperon, G. E., Burlakov, V. M., Docampo, P., Goriely, A. \& Snaith, H. J. Morphological control for high performance, solution-processed planar heterojunction perovskite solar cells. Adv. Funct. Mater. 24, 151-157 (2014)

27. Wong, C. Y., Folie, B. D., Cotts, B. L. \& Ginsberg, N. S. Discerning variable extents of interdomain orientational and structural heterogeneity in solutioncast polycrystalline organic semiconducting thin films. J. Phys. Chem. Lett. 6, 3155-3162 (2015).

28. Guo, Z., Manser, J. S., Wan, Y., Kamat, P. V. \& Huang, L. Spatial and temporal imaging of long-range charge transport in perovskite thin films by ultrafast microscopy. Nat. Commun. 6, 7471-7478 (2015).

29. Schnedermann, C. et al. Sub-10 fs time-resolved vibronic optical microscopy. J. Phys. Chem. Lett. 7, 4854-4859 (2016).

30. Butler, S. Z. et al. Progress, challenges, and opportunities in two-dimensional materials beyond graphene. ACS Nano 7, 2898-2926 (2013).

31. Dostál, J., Pšenčík, J. \& Zigmantas, D. In situ mapping of the energy flow through the entire photosynthetic apparatus. Nat. Chem. 8, 705-710 (2016). 
32. Fuller, F. D. \& Ogilvie, J. P. Experimental implementations of twodimensional fourier transform electronic spectroscopy. Annu. Rev. Phys. Chem. 66, 667-690 (2015)

33. Wagner, W., Li, C., Semmlow, J. \& Warren, W. S. Rapid phase-cycled twodimensional optical spectroscopy in fluorescence and transmission mode. Opt. Express 13, 3697-3706 (2005).

34. Tekavec, P. F., Lott, G. A. \& Marcus, A. H. Fluorescence-detected twodimensional electronic coherence spectroscopy by acousto-optic phase modulation. J. Chem. Phys. 127, 214307-214327 (2007).

35. Draeger, S., Roeding, S. \& Brixner, T. Rapid-scan coherent 2D fluorescence spectroscopy. Opt. Express 25, 3259-3267 (2017).

36. De, A. K., Monahan, D., Dawlaty, J. M. \& Fleming, G. R. Two-dimensional fluorescence-detected coherent spectroscopy with absolute phasing by confocal imaging of a dynamic grating and 27-step phase-cycling. J. Chem. Phys. 140, 194201-194208 (2014).

37. Ostrander, J. S., Serrano, A. L., Ghosh, A. \& Zanni, M. T. Spatially resolved two-dimensional infrared spectroscopy via wide-field microscopy. ACS Photonics 3, 1315-1323 (2016).

38. Baiz, C. R., Schach, D. \& Tokmakoff, A. Ultrafast 2d ir microscopy. Opt. Express 22, 18724-18735 (2014).

39. Goetz, S., Li, D., Kolb, V., Pflaum, J. \& Brixner, T. Coherent two-dimensional fluorescence micro-spectroscopy. Opt. Express 26, 3915-3925 (2018).

40. Blair, D. P. \& Sydenham, P. H. Phase sensitive detection as a means to recover signals buried in noise. J. Phys. E. 8, 621-627 (1975).

41. Zhang, T., Borca, C. N., Li, X. \& Cundiff, S. T. Optical two-dimensional fourier transform spectroscopy with active interferometric stabilization. Opt. Express 13, 7432-7441 (2005).

42. Scheuring, S. \& Sturgis, J. N. Chromatic adaptation of photosynthetic membranes. Science 309, 484-487 (2005).

43. Moulisová, V. et al. Low light adaptation: energy transfer processes in different types of light harvesting complexes from rhodopseudomonas palustris. Biophys. J. 97, 3019-3028 (2009).

44. Southall, J. et al. Characterisation of a pucBA deletion mutant from rhodopseudomonas palustris lacking all but the pucBAd genes. Photo. Res. 135, 9-21 (2018).

45. Brotosudarmo, T. H. P. et al. Single-molecule spectroscopy reveals that individual low-light LH2 complexes from rhodopseudomonas palustris 2.1.6. have a heterogeneous polypeptide composition. Biophys. J. 97, 1491-1500 (2009).

46. Bischak, C. G. et al. Origin of reversible photoinduced phase separation in hybrid perovskites. Nano. Lett. 17, 1028-1033 (2017).

47. Dahlberg, P. D. et al. Mapping the ultrafast flow of harvested solar energy in living photosynthetic cells. Nat. Commun. 8, 988-994 (2017)

48. Mertz, J. Introduction to Optical Microscopy.. (Roberts and Company Publishers, Greenwood Village, 2009).

49. Novotny, L. \& van Hulst, N. Antennas for light. Nat. Photonics 5, 83-90 (2011).

50. Karvtsov, V., Ulbricht, R., Atkin, J. M. \& Raschke, M. B. Plasmonic nanofocused four-wave mixing for femtosecond near-field imaging. Nat. Nanotechnol. 11, 459-464 (2016).

51. Yampolsky, S. et al. Seeing a single molecule vibrate through time-resolved coherent anti-stokes raman scattering. Nat. Photonics 8, 650-656 (2014).

52. Penwell, S. B., Ginsberg, L. D. S. \& Ginsberg, N. S. Bringing far-field subdiffraction optical imaging to electronically coupled optoelectronic molecular materials using their endogenous chromophores. J. Phys. Chem. Lett. 6, 2767-2772 (2015)

53. Yetzbacher, M. K., Belabas, N., Kitney, K. A. \& Jonas, D. M. Propagation, beam geometry, and detection distortions of peak shapes in two-dimensional fourier transform spectra. J. Chem. Phys. 126, 044511-044529 (2007).

54. Ulness, D. J., Kirkwood, J. C. \& Albrecht, A. C. Competitive events in fifth order time resolved coherent raman scattering: direct versus sequential processes. J. Chem. Phys. 108, 3897-3902 (1998).

55. Blank, D. A., Kaufman, L. J. \& Fleming, G. R. Fifth-order two-dimensional Raman spectra of CS2 are dominated by third-order cascades. J. Chem. Phys. 111, 3105-3114 (1999).

56. Senlik, S. S., Policht, V. R. \& Ogilvie, J. P. Two-color nonlinear spectroscopy for the rapid acquisition of coherent dynamics. J. Phys. Chem. Lett. 6, 2413-2420 (2015).

57. Donnert, G., Eggeling, C. \& Hell, S. W. Major signal increase in fluorescence microscopy through dark-state relaxation. Nat. Methods 4, 81-86 (2007).

58. Moerner, W. E. \& Fromm, D. P. Methods of single-molecule fluorescence spectroscopy and microscopy. Rev. Sci. Instrum. 74, 3597-3619 (2003).

59. Hildner, R., Brinks, D., Nieder, J. B., Cogdell, R. J. \& van Hulst, N. F. Quantum coherent energy transfer over varying pathways in single light-harvesting complexes. Science 340, 1448-1451 (2013).

60. Ferretti, M. et al. Dark states in the light harvesting complex 2 revealed by two-dimensional electronic spectroscopy. Sci. Rep. 6, 20834-20842 (2016).
61. Harel, E. \& Engel, G. S. Quantum coherence spectroscopy reveals complex dynamics in bacterial light-harvesting complex 2 (LH2). Proc. Natl Acad. Sci USA. 109, 706-711 (2012).

62. Lott, G. A. et al. Conformation of self-assembled porphyrin dimers in liposome vesicles by phase-modulation 2D fluorescence spectroscopy. Proc. Natl Acad. Sci. USA 108, 16521-16526 (2011).

63. Mastron, J. N. \& Tokmakoff, A. Fourier transform fluorescence-encoded infrared spectroscopy. J. Phys. Chem. A 122, 554-562 (2017).

64. Thyrhaug, E., Žídek, K., Dostál, J., Bína, D. \& Zigmantas, D. Exciton structure and energy transfer in the Fenna-Matthews-Olson complex. J. Phys. Chem. Lett. 7, 1653-1660 (2016).

65. Hess, S., Akesson, E., Cogdell, R. J., Pullerits, T. \& Sundström, V. Energy transfer in spectrally inhomogeneous light-harvesting pigment-protein complexes of purple bacteria. Biophys. J. 69, 2211-2225 (1995).

66. Niedzwiedzki, D. M. et al. Spectroscopic studies of two spectral variants of light-harvesting complex 2 (LH2) from the photosynthetic purple sulfur bacterium Allochromatium vinosum. Biochim. Biophys. Acta Bioenerg. 1817, 1576-1587 (2012).

67. Karki, K. J. et al. Unexpectedly large delocalization of the initial excitation in photosynthetic light harvesting. Preprint at https://arxiv.org/abs/1804.04840 (2018).

68. Nardin, G., Autry, T. M., Silverman, K. L. \& Cundiff, S. T. Multidimensiona coherent photocurrent spectroscopy of a semiconductor nanostructure. Opt. Express 21, 28617-28627 (2013).

69. Martin, E. W. \& Cundiff, S. T. Inducing coherent quantum dot interactions. Phys. Rev. B 97, 081301-081305 (2018).

70. Simpson, M. J., Doughty, B., Yang, B., Xiao, K. \& Ma, Y.-Z. Imaging electronic trap states in perovskite thin films with combined fluorescence and femtosecond transient absorption microscopy. J. Phys. Chem. Lett. 7, 1725-1731 (2016)

\section{Acknowledgements}

The authors acknowledge the AFOSR Biophysics program for support of this research under grant FA9550-15-1-0210. R.J.C. and A.T.G. gratefully acknowledge support from the Photosynthetic Antenna Research Center, an Energy Frontier Research Center funded by the U.S. Department of Energy, Office of Science, Office of Basic Energy Sciences under Award Number DE-SC 0001035. The authors also thank Julia Widom, Andrew Marcus, and Eric Martin, Travis Autry of the Cundiff group for the enthusiastic help in building the fluorescence-based 2D setup, and Damon Hoff for advice regarding the microscope setup and sample preparation.

\section{Author contributions}

J.P.O. conceived the idea for the method. V.T. and Y.A.M designed the experiment in consultation with J.P.O. R.J.C., and A.T.G. gave advice on the sample choice and preparation and provided the samples. V.T. and Y.A.M. performed the experiments and data analysis. T.L.C.J. performed comparative analysis of signal contributions in conventional and F-2DES. V.T., Y.A.M., and J.P.O. wrote the paper with input from all of the authors

\section{Additional information}

Supplementary Information accompanies this paper at https://doi.org/10.1038/s41467018-06619-x.

\section{Competing interests: The authors declare no competing interests.}

Reprints and permission information is available online at http://npg.nature.com/ reprintsandpermissions/

Publisher's note: Springer Nature remains neutral with regard to jurisdictional claims in published maps and institutional affiliations.

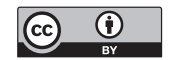

Open Access This article is licensed under a Creative Commons Attribution 4.0 International License, which permits use, sharing, adaptation, distribution and reproduction in any medium or format, as long as you give appropriate credit to the original author(s) and the source, provide a link to the Creative Commons license, and indicate if changes were made. The images or other third party material in this article are included in the article's Creative Commons license, unless indicated otherwise in a credit line to the material. If material is not included in the article's Creative Commons license and your intended use is not permitted by statutory regulation or exceeds the permitted use, you will need to obtain permission directly from the copyright holder. To view a copy of this license, visit http://creativecommons.org/ licenses/by/4.0/.

(C) The Author(s) 2018 